\title{
Le récit en questions : introduction
}

\section{André Petitjean}

\section{OpenEdition}

\section{Journals}

Édition électronique

URL : http://journals.openedition.org/pratiques/5598

DOI : 10.4000/pratiques.5598

ISSN : 2425-2042

\section{Éditeur}

Centre de recherche sur les médiations (CREM)

Référence électronique

André Petitjean, «Le récit en questions : introduction », Pratiques [En ligne], 181-182 | 2019, mis en ligne le 17 juin 2019, consulté le 24 septembre 2020. URL : http://journals.openedition.org/pratiques/ 5598 ; DOI : https://doi.org/10.4000/pratiques.5598

Ce document a été généré automatiquement le 24 septembre 2020

(c) Tous droits réservés 


\title{
Le récit en questions : introduction
}

\author{
André Petitjean
}

1 Ce numéro de Pratiques a été conçu en fonction d'une double logique, à la fois historique et synchronique. Pour la première, comme l'attestent les différents numéros de Pratiques, depuis la création de la revue en 1974, des théorisations narratives diverses ont été défendues au fil des différentes livraisons sur lesquelles il importe de porter un regard rétrospectif. Pour la seconde, elle correspond au fait que le numéro s'inscrit dans le programme de recherche du Centre de recherche sur les médiations (Crem) intitulé «Narrations de la société/sociétés de la narration » tel qu'il est consacré au récit et aux différentes formes de narration sociale. L'enjeu est de faire interagir des recherches qui mettent l'accent sur la description et la classification avec d'autres plus spéculatives et interprétatives.

2 Le numéro s'organise autour de trois axes. Une première partie ( Les théories du récit en débat ») confronte certains paradigmes du récit, privilégiant la linguistique textuelle et discursive (J.-M. Adam, A. Rabatel), la sémiotique narrative (D. Bertrand, M. Colas-Blaise), l'ethnocritique (J.-M. Privat), la perspective cognitiviste (A. Fragonara). Une attention particulière a été réservée aux récits, fictionnels (S. Patron et B. Richardson) ou non (F. Revaz), dont l'existence nécessite l'invention de nouvelles méthodes et un outillage narratologique adapté pour en rendre compte. Ce faisant, il ne nous a pas semblé utile de revenir sur le regain attesté de la narratologie dans les débats actuels des sciences humaines en France et au-delà de l'hexagone. On peut se référer, à ce propos, au tableau que dresse R. Baroni (2016) dans la $30^{\mathrm{e}}$ livraison de Questions de communication et aux articles qui, dans le $\mathrm{n}^{\circ} 31$ de la même revue (Baetens, 2017 ; Fleury \& Walter, 2017; Jost, 2017 ; Rabatel, 2017; Saurier \& Vallée, 2017 ; Schmitt, 2017), ont nourri, en forme de réponse, un débat fort intéressant. On se reportera aussi, pour mesurer le nombre d'ouvrages et d'articles relevant de la narratologie, aux revues Poetics Today, Vox poetica, Cahiers de narratologie. Dans cette première partie, il n'est pas non plus question de traiter frontalement certains concepts narratologiques fondamentaux, comme le fait par exemple S Patron $(2009,2015)$ mais de faire interagir différentes disciplines. 
3 C'est ainsi que Jean-Michel Adam commence par discuter du bien fondé de l'opposition entre narratologie classique et post-classique. La première, dont les représentants sont R. Barthes, G. Genette, C. Bremond, A. J. Greimas, T. Todorov, serait entachée de structuralisme et privilégierait les corpus littéraires. La seconde, au contraire, serait pluridisciplinaire, plus ouverte, tant sur le plan méthodologique que des objets d'études, et susceptible de rendre compte de l'inflation du fait narratif. Ce que conteste J.-M. Adam en rappelant que dès ses origines la narratologie se concrétise par la présence de chercheurs issus de domaines différents travaillant sur des objets de discours qui ne sont pas que littéraires. J.-M. Adam se livre ensuite à une relecture du cadre épistémologique des théorisations narratives de l'époque en montrant en particulier comment la narratologie de R. Barthes, dans la mouvance d'É. Benveniste, prend appui sur ce qu'il appelle la «translinguistique » selon une filiation bien plus rhétorique que poétique. Finalement, J.-M. Adam défend une approche linguistique de la narration dès lors qu'elle se donne les moyens de penser les niveaux d'intégration des unités d'un texte et de différencier les différents types textuels et genres de discours.

4 Renouant avec les grands principes aristotéliciens et le retour au premier plan des émotions, dans la lignée des travaux pluridisciplinaires les plus récents, Alain Rabatel consacre sa contribution au phénomène de l'empathie. Dans un premier temps, prenant soin de distinguer, même si la chose n'est pas aisée, sympathie et empathie, il examine les différents modes de son actualisation. Plus précisément, il montre que sur son versant linguistique, l'empathie consiste à se mettre à la place des autres, à envisager de leur point de vue ce qu'ils perçoivent, ressentent, pensent, disent ou font. Dans un second temps, A. Rabatel développe la notion de mobilité empathique qui consiste à s'intéresser aux autres, dans leur diversité, en essayant de les comprendre de l'intérieur, depuis les situations, les expériences, les valeurs et les connaissances qui sont les leurs. Dans la mesure où la mobilité empathique peut s'appliquer à différents supports d'empathie, figuratifs ou abstraits, on mesure combien les récits représentent un terrain d'expériences multiples (somesthésique, esthétique, éthique, sociopolitique).

5 Denis Bertrand constate lui aussi l'essor de la narratologie dans les débats actuels des sciences humaines en France mais il s'étonne de l'occultation de la «sémiotique narrative et discursive » greimasienne, comme le fait R. Baroni (2016, p. 220) qui écrit dans l'article précédemment cité : «Certes, on croise encore, ici ou là, quelques greimasiens qui s'accrochent à leur radeau sémiotique ». Désireux de montrer que ce paradigme est loin d'être en voie d'extinction, D. Bertrand entreprend d'en faire l'historique et de reconstituer les fondements conceptuels de ce champ théorique. Ce faisant, il reconnait qu'au fil du temps le récit et la narratologie n'ont plus été au centre des préoccupations sémiotiques. D. Bertrand montre, ensuite, en quoi les travaux des épigones d'A. J. Greimas, en particulier ceux consacrés aux problématiques de l'émotion et de la passion, associées à celle de la narrativité, sont susceptibles d'enrichir les théories du récit. Le bilan est d'autant plus convaincant que D. Bertrand a l'art d'illustrer son propos par des analyses ponctuelles d'extraits littéraires d'époques diverses.

6 Marion Colas-Blaise, de son côté, revendique, elle aussi, son attachement au cadre théorique de la sémiotique greimasienne et post-greimasienne, dans le but de confirmer le caractère structurant du récit comme modèle d'intelligibilité. Elle le 
prouve en s'appliquant à analyser une image fixe, en l'occurrence des tableaux de P. Klee et pour ce faire élabore une modélisation qui intègre à la sémiotique des éléments d'herméneutique, mais aussi de sociologie et d'anthropologie. Il s'agit pour M. Colas-Blaise d'apporter des réponses à des questions du type l'image fixe à scène unique peut-elle raconter? Si oui, on doit se demander dans quelle mesure la structure narrative active des potentialités de l'image qui doivent être actualisées ou réalisées par le spectateur. Et si on va plus avant, dans quelle mesure l'art abstrait exige-t-il de repenser la notion de narrativité ? Sur ces bases, il s'agit de montrer que le point de vue de la narrativité permet de rendre compte de la capacité des "compositions cinétiques» de P. Klee à générer un savoir non seulement sur le fonctionnement de l'image " narrative », mais sur le monde dans lequel elle fait sens.

7 Aurora Fragonara, quant à elle, commence par rappeler que les analyses structurales des récits des années 1960 et 1970 ont essentiellement porté sur les principes et les outils universaux susceptibles de rendre compte de la structure fondamentale de la narration au-delà de la variété de ses manifestations. Faire un retour critique sur l'intérêt et les limites de ces théorisations sémiotiques nécessite une réflexion de type épistémologique sur l'activité même de modélisation et sur les conditions et les modalités de leur emploi. Ce que propose A. Fragonara en s'appuyant sur les philosophes des sciences et sur U. Eco. Ce dernier reproche aux modèles structuralistes leur usage essentialiste et normatif. Avançant plus avant dans sa réflexion, A. Fragonara pense avoir trouvé dans la théorie de l'énaction, issue des sciences cognitives, la possibilité de combler la fracture entre l'aspect gnoséologique et ontologique des modélisations grâce à la notion d'interaction capable d'identifier un terrain commun pour les deux points de vue, celui du producteur et celui du récepteur d'un même produit culturel. Cette conception énactée du schéma narratif permet en effet d'appréhender différemment les variations tant interculturelles qu'à l'intérieur d'un même contexte socio-culturel. Pour terminer, A. Fragonara tente d'illustrer la puissance d'une modélisation dans une perspective cognitive et énactive en l'appliquant à une étude de la structure narrative du Petit Prince.

8 Jean-Marie Privat, pour sa part, ouvre, avec son analyse de la nouvelle de J. L. Borges (1974) «La mort et la boussole », un débat direct avec R. Baroni (2003) qui s'est livré dans la revue Poétique à une étude du même récit borgésien. S'il partage avec R. Baroni le fait que la nouvelle de J. L. Borges est complexe et met le lecteur à l'épreuve de ses propres compétences génériques, il prend par contre ses distances par rapport à une lecture qui serait dominée par le formalisme narratologique. Reprochant au narratologue une insuffisante perspective anthropologique, J.-M. Privat, à partir d'une étude des principaux antagonistes du récit, montre l'existence de cas concrets d'hétérophonie culturelle. Ce qui lui permet d'analyser de quelle façon une l'approche lettrée traditionnelle fait obstacle à une interprétation anthropologique du récit, son architecture, ses valeurs et ses croyances.

9 C'est ainsi qu'il propose de ne pas se limiter à opposer terme à terme, pathos et logos, voire pathos, logos et mythos mais de recourir à un code herméneutique sous la forme d'un losange dont les diagonales mettraient en dialogie discursive et culturelle pathos/ logos/mythos/métis.

10 Pour Sylvie Patron et l'entretien qui suit avec Brian Richardson, il s'agit d'envisager le récit de manière prospective à partir de la problématique du récit «non naturel ». Ce qui doit se comprendre comme des récits "antimimétiques» qui, au niveau des 
personnages comme du monde représenté, dérogent aux conventions des genres relevant de la pratique du réalisme. Pour en rendre compte, s'est développée une narratologie non naturelle dont S.Patron tente d'en décrire les différentes orientations. Pour certains, il s'agit d'observer les modes de transgression des lois de notre monde sublunaire à l'œuvre dans ces fictions alors que pour d'autres l'accent est mis sur les stratégies "non naturalisantes» utilisées par les lecteurs lorsqu'ils sont confrontés à des événements ou des scénarios non naturels. Parmi les études les plus représentatives de la narratologie non naturelle, S. Patron retient celles qui sont consacrées au temps et à l'espace ainsi qu'à l'ontologie de la métamorphose. En prenant l'exemple de la "narration omnisciente", S. Patron achève son panorama par une discussion critique de certains attendus de la narratologie non naturelle. Quant à l'entretien, à plusieurs voix avec B. Richardson, il permet de mieux comprendre l'historique, l'intérêt et les difficultés de la narratologie non naturelle telle qu'elle s'intéresse aux textes dits postmodernes.

11 Après avoir rappelé la variété des formes sémiotiques de narrativisation et la diversité des genres de récit, Françoise Revaz commence par vanter les mérites de la narratologie post-classique qui prendrait en charge, mieux que la narratologie classique, les divers paramètres de la situation de narration, les processus dynamiques de production et de réception, les fonctions du récit, ainsi que le rôle du lecteur et de son activité d'interprétation. F. Revaz illustre ensuite son propos en prenant comme corpus, dans le cadre de la "thérapie narrative » et de "néonarrativité » groupale, l'enregistrement de récits produits lors d'entretiens psychothérapeutiques. Les caractéristiques les plus saillantes que possèdent ces récits sont leur aspect fragmentaire, non fini et épisodique. À quoi s'ajoute le fait que ces récits sont plutôt dialogiques, co-construits par le patient avec le thérapeute et les autres membres de la famille. Pour finir, F. Revaz, s'appuyant sur des exemples, interroge les critères de racontabilité et de véracité et souligne la dimension polylogale des récits étudiés.

Dans la seconde partie du numéro, ("Fictions et non fictions contemporaines»), il s'est agi de rendre compte du statut du récit dans les productions actuelles, qu'elles aient la forme de romans (S. Lawson), de pièces de théâtre (A.Petitjean), de «narrations documentaires », (C. Lacoste), de séries télévisées (M. Boni et C. Martinez) ou de jeux vidéo (D. Compagno).

13 C'est ainsi que Sophie Lawson s'intéresse à la place qu'occupent les animaux dans nombre de fictions romanesques contemporaines. Certes la présence d'animaux dans les fictions n'est pas nouvelle mais le changement qui s'opère dans l'abondant corpus que S.Lawson a rassemblé tient au fait qu'ils ne sont plus conçus comme des projections anthropomorphisées de l'humanité mais sont considérés dans leur individualité animale. Cela s'explique, pour une part, outre par les préoccupations écologiques à l'ère de l'anthropocène et des dégâts irréversibles sur le vivant, au fait que l'imaginaire fictionnel est en phase avec les avancées récentes des sciences zoologiques et de l'éthologie cognitive. Comme le montre S. Lawson, il s'agit de tenter de sortir de l'humanocentrisme et de l'anthroporéglage narratif, que le registre de ces récits emprunte à la distanciation ironique ou relève plutôt de l'élégie et du lyrisme. Dans tous les cas, le fait d'adopter un point de vue zoocentré nécessite l'utilisation de procédés (forme de la liste ou tableau descriptif) et de techniques narratives (point de vue, instance narratrice, monologues intérieurs) au service d'une intention à la fois éthique et esthétique des auteurs. 
14 André Petitjean part du constat que depuis quelques décennies, on assiste à une véritable vogue de la parole solitaire au théâtre sous la forme de pièces insérées dans des recueils ou d'œuvres autonomes. À l'examen de ces textes, il apparait qu'au niveau narratif ils sont loin de respecter l'ordonnancement de la fable selon les principes aristotéliciens (unicité de l'intrigue, progression ordonnée et finalisée des actions, développement temporel chronologique, causalité explicite des enchainements). Pour le montrer, A. Petitjean, dans un premier temps, analyse en quoi ces textes ont la forme de récits fragmentés car la fable, proche en cela des fictions romanesques, n'est plus soumise au développement temporel strictement chronologique. Grâce à une alternance entre narration et monstration, le personnage peut voyager en permanence dans des chronotopes différents, passés, présents, à venir. Dans un second temps, A. Petitjean montre que la solitude du personnage n'est pas forcément anti-dramatique puisque les monologues sont très polyphoniques. Leur intérêt est de faire advenir de nombreux personnages et c'est là un indice du processus de romanisation que connait le théâtre depuis plusieurs années.

15 Charlotte Lacoste s'intéresse à ce que d'aucuns appellent la «non-fiction", dénomination qui recouvre, au-delà de la diversité des œuvres et des auteurs, des textes qui partagent une même intention documentaire. Dans un premier temps, C. Lacoste s'emploie à caractériser ce nouveau corpus en vogue depuis une vingtaine d'années. Il apparait que ces œuvres ont une dimension documentaire mais en rupture avec les usages littéraires des documents dans les romans du XIX ${ }^{e}$ siècle. Il ne s'agit plus de les utiliser pour produire des «effets de réel » au service d'un vraisemblable fictionnel mais de s'assumer comme documents à part entière. Il s'ensuit que ces œuvres, qui privilégient la description, le fragment et la liste, prennent leur distance avec la fiction, au sens où elles remettent en question les vertus configuratrices que l'on prête ordinairement à la "mise en intrigue ». Dans un second temps, C. Lacoste illustre son propos théorique en examinant dans le détail les procédés factographiques de deux auteurs (J. Rollin et A. Ernaux) qui ont en commun de mettre en scène l'insigne quotidien de nos vies ordinaires. Dans sa conclusion, C. Lacoste insiste sur l'aspect disruptif de ces œuvres dans la mesure où elles interrogent le statut même de la Littérature.

16 De plus en plus présente notamment à la télévision et grâce aux nouveaux fournisseurs de services comme Netflix, la sérialité fait l'objet de l'article de Marta Boni et Camille Martinez qui entreprennent d'étudier certaines particularités de ces récits. Dans un premier temps, les deux auteures, rappellent l'importance de la temporalité des récits sériels, qui a fait l'objet de nombreux travaux. Dans un second temps, et c'est l'essentiel de leur recherche, M.Boni et C.Martinez, alliant dans leur étude esthétique, narratologie et pragmatique de la réception, choisissent d'analyser l'espace fictionnel des séries. En prenant appui sur un corpus étendu, elles montrent que l'espace, bien audelà de son statut de simple décor, est à considérer comme un vecteur de la narration, comme un monde meublé d'objets, de situations, de personnages et de lois naturelles. Penser les séries comme des expériences de construction de mondes, d'espaces pluridirectionnels et explorables, est l'un des mécanismes qui expliquent le succès des séries.

17 L'importance prise par les jeux vidéo dans les pratiques culturelles de nos sociétés est incontestable. Afin de comprendre la portée du phénomène, Dario Compagno fait l'hypothèse que les jeux vidéo offrent à leurs usagers la possibilité de faire l'expérience 
de la liberté autrement que dans les actions de la vie quotidienne. Pour en rendre compte, D. Compagno commence par comparer le modèle de l'action que propose E. Anscombe à celui de l'action racontée développé par A.-J. Greimas en soulignant, outre leur incompatibilité, leur incapacité de rendre compte des actions interactives que présupposent les jeux vidéo. D. Compagno a alors recours à deux modèles explicatifs, l'un qu'il emprunte à la vision du monde d'A. Schopenhauer, l'autre à la pensée sémiotique d'U. Eco. Ce qui lui permet de montrer qu'analyser un jeu vidéo demande de développer une grammaire de l'action capable de décrire comment un sens se produit dans l'interaction entre d'un côté une histoire pré-formée et de l'autre la vraie responsabilité du joueur.

Quant à la troisième partie ("Les récits en situation scolaire ») sa place relativement restreinte s'explique par le fait que le récit a fait l'objet de nombreux numéros spéciaux de la revue : les numéros 11-12 (1976), 14 (1977) pour des approches narratologiques ou 133-134 (2007) pour le traitement du récit en situation scolaire selon les disciplines. Ce serait l'objet d'un article à part entière que d'analyser la place du récit dans Pratiques à travers de multiples objets (l'activité de raconter, les genres du récit, le personnage, les dialogues romanesques, les temps verbaux, les fables de La Fontaine, etc.). Il reste que pour l'essentiel, les théories du récit ont servi, après leur transposition, à élaborer de l'enseignable susceptible de développer les compétences scripturales et lecturales en matière de récit, des élèves des premier et second degrés. Cette double approche compte de nombreux articles.

Pour sa part, Yves Reuter, après avoir expliqué en quoi les didactiques sont des disciplines de recherche à part entière, rappelle que les récits sont présents dans la totalité des disciplines scolaires. Il explique le phénomène en relevant les nombreuses fonctions, tant didactiques que pédagogiques, qui sont celles des récits en situation d'enseignement. Pour illustrer son propos, Y. Reuter s'appuie sur une recherche au cours de laquelle des consignes d'écriture sollicitant des récits aux contenus différents (les uns faisant référence à du vécu les autres à l'imaginaire) ont été données aux élèves. L'auteur s'attache à montrer en quoi ces consignes influencent le contenu des récits produits par les élèves. L'analyse porte essentiellement sur des classes relevant de la pédagogie Freinet avec en arrière-plan des allusions comparées à la pédagogie traditionnelle. Observer les récits en prenant en compte les contenus est une tâche problématique compte tenu de la multiplicité des paramètres. C'est pourquoi Y. Reuter se limite à deux éléments majeurs : i) les récits fondés sur le vécu et une certaine conception de la vérité ; ii) la place accordée aux dialogues. Pour la vérité, les élèves ont bien du mal d'échapper à leur désir d'être fidèles aux événements qu'ils racontent tout en ayant conscience que les lois du récit font qu'ils doivent opérer des choix étant donné les contraintes de mise en forme. À quoi s'ajoute la difficulté de rendre compte d'une expérience intime dans le cadre de l'espace public de l'école. Concernant les dialogues, les élèves ayant intériorisé la nécessité d'éviter la banalité des propos résiduels, finissent par se montrer trop économes. Pour terminer, Y. Reuter montre que les contenus des récits produits, au-delà des critères textuels sont aussi dépendants des modalités d'enseignement et des positions d'apprentissage liées de la configuration disciplinaire.

On sait que les pratiques scolaires sont pourvoyeuses de nombreux écrits (cahier de textes, «cahier de roulement », classeur d'élève, etc.) qui en gardent la trace. Aurore Promonet s'attache à étudier ce genre d'écrits en se demandant si on peut les assimiler 
à des récits et si oui quelles en sont les fonctions. Le potentiel narratif de ces écrits tient au fait qu'ils racontent la vie de la classe selon une chronologie liée au temps scolaire sous la responsabilité des enseignants. Dans un premier temps, précisant que ces écrits prennent aujourd'hui des formes diverses (cahier, carnet du lecteur, cahier d'expérience, journal d'apprentissage, écrits d'appropriation), A. Promonet s'emploie à définir le statut commun de ces écrits, tant d'un point de vue institutionnel, pédagogique, didactique que discursif. Elle montre, en particulier que les cahiers présentent deux dimensions mémorielles, combinant la mémoire affective de l'enfant et la mémoire cognitive du sujet didactique. Dans un second temps, A. Promonet rend compte, dans le cadre d'une recherche-action au sein du LéA TEC ${ }^{1}$, du fonctionnement $\mathrm{du}$ « journal des apprentissages » réalisé, quotidiennement et tout au long de l'année, dans une classe de CM2. Elle en souligne l'intérêt, le journal constituant un récit de l'expérience scolaire d'apprentissage, oscillant entre un rendu objectif et l'expression subjective.

21 Pierre Moinard, de son côté, s'intéresse aux rapports qu'entretiennent les lecteurs adolescents à la lecture des récits de fiction. Pour ce faire, il s'appuie sur un corpus constitué à partir du site forum-ados.fr dont l'une des rubriques (" société et culture »), est consacrée aux débats sur les lectures de livres. Dans un premier temps, P. Moinard précise son cadre de référence en matière de lecture de la fiction (J.-L. Dufays pour la dialectique participation/distanciation, J.-M.Schaeffer pour "l'immersion fictionnelle» et G. Langlade pour la notion d'«activité fictionnalisante du sujet lecteur »). Ensuite il élabore un protocole d'analyses qui le conduit à distinguer différents types d'expériences de lecture de récits échangées. Ce qui le conduit à affirmer que le site forum-ados joue le rôle d'un réseau social utile pour lire plus, et plus intensément tout en optimisant les joies de l'immersion dans les récits. Dans une dernière partie, en s'appuyant sur un corpus de billets produits par des élèves de $3^{\mathrm{e}}, 2^{\mathrm{e}}$ et $1^{\text {re }}, \mathrm{P}$. Moinard défend l'intérêt de mener des études portant sur des échanges asynchrones entre lecteurs en contexte scolaire, en particulier quand ces billets concernent l'appropriation par les élèves d'œuvres patrimoniales.

Pour ne pas conclure, je dirais qu'en continuité et en rupture avec un certain structuralisme narratif, la narratologie connait depuis quelques années d'autres développements dont ce numéro aura essayé de donner un aperçu.

\section{BIBLIOGRAPHIE}

BAETENS, J. (2017). « Nouvelle narratologie, nouveau récit ». Questions de communication 31, p. 231-243.

BARONI, R. (2003). « Genres littéraires et orientation de la lecture. Une lecture modèle de La mort et la boussole de J. L. Borges ». Poétique 134, p. 141-157. En ligne : https://www.cairn.info/revuepoetique-2003-2-page-141.htm. 
BARONI, R. (2016). «L'empire de la narratologie, ses défis et ses faiblesses ». Questions de communication 30, p. 219-238. En ligne : https://www.cairn.info/revue-questions-decommunication-2016-2-page-219.htm.

BORGES, J. L. (1974) [1942]. « La mort et la boussole ». Trad. de l'espagnol par P. Verdevoye. In : Fictions. Paris : Gallimard, p. 149-163.

FLEURY, B. \& WALTER, J. (2017). « La narratologie dans tous ses états ». Questions de communication 31, p. 183-197. En ligne : https://journals.openedition.org/questionsdecommunication/11109. JOST, F. (2017). « À quelles conditions est-il possible de faire une narratologie comparée ?». Questions de communication 31, p. 265-278.

MARTI, M. (2017). « De la narratologie littéraire à la narratologie générale : l'empire et ses limes ». Questions de communication 31, p. 199-214.

PATRON, S. (2009). Le Narrateur. Introduction à la théorie narrative. Paris : Armand Colin.

PATRON, S. (2015). La Mort du narrateur et autres essais. Limoges : Lambert-Lucas.

PRATIQUES 11-12 (1976). « Récit (1) ». En ligne : https://www.persee.fr/issue/

prati_0338-2389_1976_num_11_1.

PRATIQUES 133-134 (2007). « Récits et disciplines scolaires ». En ligne : https://www.persee.fr/

issue/prati_0338-2389_2007_num_133_1.

PRATIQUES 14 (1977). « Récit (2)». En ligne : https://www.persee.fr/issue/

prati_0338-2389_1977_num_14_1.

RABATEL, A. (2017). « Des récits en général, de la narratologie en particulier ». Questions de communication 31, p. 245-263.

SAURIER, D. \& VALLÉE, O. (2017). « Récits, figures et chemins de traverse ». Questions de communication 31, p. 279-290.

schмiтт, A. (2017). « La pratique narratologique ». Questions de communication 31, p. 215-229.

\section{NOTES}

1. Lieu d'éducation associé à l'Institut français de l'éducation «Traces écrites en terres de Lorraine » (LéA TEC).

\section{AUTEUR}

\section{ANDRÉ PETITJEAN}

Université de Lorraine, Crem, F-57000 Metz, France 